\title{
Magnetic nanoparticles for magnetically guided therapies against neural diseases
}

\author{
G.F. Goya, M.P. Calatayud, B. Sanz, M. Giannaccini, V. Raffa, \\ T.E. Torres, and M.R. Ibarra
}

Neurological pathologies and nerve damage are two problems of significant medical and economic impact because of the hurdles of losing nerve functionality in addition to significant mortality and morbidity, and demanding rehabilitation. There are currently a number of examples of how nanotechnology can provide new solutions for biomedical problems. Current strategies for nerve repair rely on the use of functionalized scaffolds working as "nerve guidance channels" to improve axonal regeneration and to direct axonal re-growth across the nerve lesion site. Since low invasiveness and high selectivity of the growth stimulation are usually conflicting requirements, new approaches are being pursued in order to overcome such limitations. Engineered magnetic nanoparticles (MNPs) have emerged from this need of noninvasive therapies for both positioning and guiding neural cells in response to an external magnetic field. Here, we review the current state of the use of MNPs for neuroprotective and magnetically guided therapies. We discuss some conceivable outcomes of current magnetically driven strategies seeking integrated platforms for regenerative action on damaged tissues.

\section{Introduction}

A nerve in the peripheral nervous system is a cord-like structure that contains many axons. It includes neurons and nonneuronal Schwann cells that coat the axons in myelin. Myelin is an electrically insulating material and forms a sheath around the axon, which is fundamental for the proper conduction and transmission of electrochemical impulses, by insulating the axons from electrically charged atoms and molecules. Complete nerve injury (neurotmesis) results in the death of both distal axons and Schwann cells (i.e $\equiv$ se cells respensible to supply the myelin for peripherans) with consequent functional loss in innervated organs. In contrast to the central nervous system, the peripheral nervous system has relevant regenerative capability, although complete functional recovery is rarely reached. There are a large number of different cell types involved in the healing and regeneration of an injured nerve; for example, in a peripheral nerve lesion, the axon distal from the injury site degenerates, and Schwann cells and later macrophages clean the neural tubes of cell debris and myelin, so-called Wallerian degeneration. ${ }^{1} \mathrm{M} \equiv$ is a layer of membranous structure around axons respensible for the fast and efficient propagation of electrieal impulses through the neural system.

When an injury occurs, a gap between the nerve's damaged ends is produced, and surgical intervention is necessary. Nowadays, there are two main strategies for those injuries with a long gap. In the first strategy, synthetic or biological conduits are sutured to each stump in order to target the distal end, avoiding scar tissue infiltration. A second approach to long gaps uses autologous nerve grafts (autografts) to provide a natural guidance channel populated with functioning Schwann cells, but this is challenging due to donor site collateral effects and patient condition. ${ }^{2,3}$ Because the axon regrows quite slowly (about $2-5 \mathrm{~mm}$ per day), it is extremely important to accelerate 
the regeneration time or to expand the time window of opportunity. To reach these goals, current experimental strategies include the usage of neurotrophins (proteins that regulate the development, maintenance, and function of neurons), growth factors, neurotransmitters, extracellular matrix proteins, and cell therapies mainly based on the use of Schwann cells and mesenchymal stem cells. ${ }^{4}$

In the last decade, we have seen nanoscience gain increasing control over the design, synthesis, and application of nanomaterials to nanodevices, starting what we recognize as nanotechnology. Nanostructured materials constitute a set of potential new therapies for neuroprotective and neuroregenerative purposes. These new therapies based on nanotechnology will require the ability to fabricate, characterize, and functionalize a variety of nano-objects adapted to each specific need. ${ }^{5,6}$ One main purpose of these bio-nanotechnological approaches is to transform current scaffold strategies into a more precise way to control both physical and biological signals promoting nerve regeneration. ${ }^{7}$ Furthermore, as these materials provide new tools for neural therapies, they inspire new ways to improve care for patients with nervous system disorders. ${ }^{8}$ This review provides a general overview of how the magnetic properties of magnetic nanoparticles (MNPs) has been recently exploited in this field.

\section{Magnetic forces}

The physical basis of remote (magnetic) actuation requires the use of a magnetic, "pulling" agent to interact with (i.e., the magnetic nanoparticles). When placed in a magnetic field $\mathbf{B}$, the energy of a magnetic nanoparticle with magnetic (dipole) moment $\mathbf{m}$ is given by $U=-\mathbf{m} \cdot \mathbf{B}$, from which the force on the MNP performed by the field can be extracted:

$$
\mathbf{F}=\nabla(\mathbf{m} \cdot \mathbf{B})
$$

It follows from Equation 1 that the force has a maximum value along the direction of the field gradient. For decades, there have been efforts to use the principle contained in Equation 1 to generate magnetic forces capable of moving small (micron- or nano-sized, depending on the available technologies at each time) magnetic objects. The biological interest in this property resulted, for example, in highly efficient protocols used routinely for magnetic cell separation. There are many theoretical calculations on the minimum values of magnetic forces that should be generated in order to manipulate $\mathrm{N} \overline{\overline{\bar{\nabla}}}$ thin the cytoplasm. However, these estimations are based on "nude" particles and an opsonization process in a biological medium. In this process, the particles are coated with different components (e.g., albumin, IgG, apolipoproteins) of the biological medium, which change their hydrodynamic size, making the required forces much larger.

There is consensus that magnetic forces in the range of 0.1 to $1 \mathrm{pN}$ are enough to move MNPs through a moderately dense biological medium. Previous reports on magnetic manipulation have shown successful actuation in 2D systems using forces of 8-16 pN..$^{9,10}$ Also, recent experiments on magnetically guided neural growth have shown that field gradients of $45 \mathrm{~T} / \mathrm{m}$ are enough to obtain positive results regarding in vitro neural orientation. ${ }^{7}$ Direct physiological effects from magnetic forces have also been reported. It has been recently proposed that mechanical stress can be produced by magnetic forces on cell structures. For example, the production of highly reactive ions and "free radicals" involving oxygen molecules, reactive oxygen species (ROS), can decrease the cell activity. ${ }^{11}$

At room temperature, mechanical effects from thermal energy on nanometer-sized MNPs are relevant. The thermal energy $k_{\mathrm{B}} T$ (where $k_{\mathrm{B}}$ is the Boltzmann constant and $T$ is the temperature) affects the magnetic behavior of MNPs due to the strong dependence of the magnetic relaxation time $\tau_{\mathrm{N}}$ (Néel relaxation) on the particle's volume $V$ through Arrhenius exponential decay. For example, a typical 30-nm magnetite MNP (i.e., diameter $d=30 \mathrm{~nm}, T=300 \mathrm{~K}$, effective magnetic anisotropy $\left.K_{\text {eff }}=1.1 \times 10^{4} \mathrm{~J} / \mathrm{m}^{3}\right)$ is expected to display a relaxation time $\tau_{\mathrm{N}}=2 \times 10^{6} \mathrm{~s}(\sim 24$ days $)$, and thus for any practical purpose, its magnetic moment is blocked. On the other side, similar MNPs having $d=10 \mathrm{~nm}$ will relax within $\tau_{\mathrm{N}}=4.0 \times 10^{-10} \mathrm{~s}$, and so for almost any experimental measuring technique, the magnetization will average to zero in the absence of an applied magnetic field. In the latter situation, it is said that the MNPs are in their superparamagnetic (SPM) state. The SPM state of a single domain particle implies null values of both the remanence $M_{\mathrm{R}}$ (i.e., magnetization value without magnetic field) and the coercive field $H_{\mathrm{C}}$.

\section{MNPs and cell interactions}

At the cellular level, the interactions between nanomaterials and the neural system allowed us to understand many basic mechanisms of neural diseases, ${ }^{12}$ and in some cases, some new ways to repair, replace, or augment the nervous system function. ${ }^{13}$ For any specific application in biomedical sciences, the selection of MNPs is often determined by the physical properties of the magnetic material of the core constituting them, as well as the chemical/biological characteristics of the functional coating. Because they have already been approved for human use, iron-oxide NPs have been the most widely employed nanomaterial in biomedical studies so far. The magnetic cores possess the ability to remotely respond to external magnetic fields; this capacity has been exploited to manipulate NPs under external magnetic forces for targeting purposes, as well as for cell separation and labeling, drug delivery, and magnetic resonance imaging (MRI).${ }^{14}$ Moreover, the additional property of MNPs to absorb energy from time-varying magnetic fields has been used to induce cell death through heat generation in a new protocol known as magnetic hyperthermia. ${ }^{15}$

Magnetic NPs can be produced by a number of physical and chemical methods that determine the final properties of the product (see the November 2013 issue of MRS Bulletin on "Magnetic Nanoparticles"). These properties are defined by the nanoparticle shape and size, the size distribution, and the surface chemistry of the resulting particles. The final magnetic 
properties of an ensemble of colloidal MNPs are strongly influenced by the degree of structural defects or impurities of the particle core and surface. ${ }^{16}$ Synthesis methods described for iron-oxide NPs include co-precipitation, oxidative hydrolysis, microemulsions, and sol-gel reactions. ${ }^{16,17}$ The effective use of MNPs for therapeutic purposes in biomedicine requires the use of a colloidal formulation. Because MNPs tend to aggregate due to their large surface-to-volume ratio and dipole-dipole interactions, ${ }^{18}$ colloidal stabilization by surface modification is required. Additional key requirements are biocompatibility of MNPs and chemical functionality for attachment of specific ligands or functional groups. ${ }^{19,20}$

\section{MNPs for neural guidance}

The potential of magnetic materials for a non-invasive approach for nerve regeneration is related to the possibility of remote actuation of MNPs using an external magnetic field. Specifically, MNPs can be forced along the direction of the external magnetic field to induce projections of the cell body (called neurites) along the preferential direction. To improve the stability and biocompatibility, these kinds of MNPs can be coated with polymers, such as poly-L-lysine (PLL), which has been checked for the human neuroblastoma SH-SY5Y cell line and primary Schwann cell cultures of the peripheral nervous system, ${ }^{21}$ or polyethyleneimine (PEI) that was tested on neural models such as PC12 or SH-SY5Y cell lines. ${ }^{22}$

Transmission electron microscopy (TEM) has been used to demonstrate that for these neural cell types, MNPs locate in the intracellular fluid (cytosol) irrespective of the surface composition (see Figure 1), but the amount of MNP uptake for a given surface composition is characteristic of each neural cell type. This fact has been exploited in ex vivo studies on olfactory ensheathing cells (OECs) to remotely control the migration for regeneration of spinal cord injuries. ${ }^{23}$ Multimodal iron-oxide NPs have been obtained by conjugation of the NPs with Rhodamine-B (MION-Rh) and have been studied in vitro
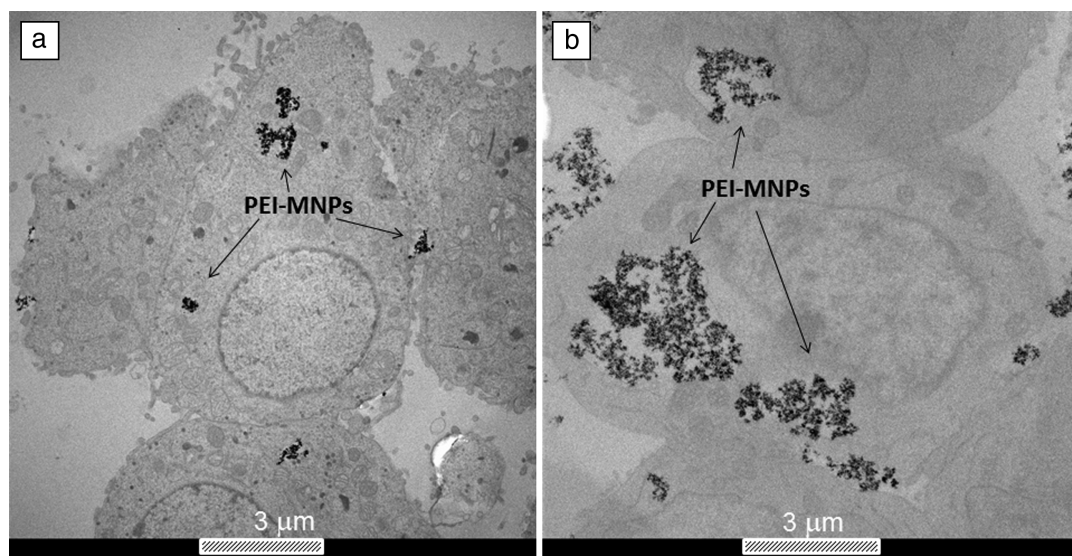

Figure 1. Typical transmission electron microscopy images of (a) PC12 and (b) SHSY5Y cells incubated with polyethyleneimine (PEI)-coated magnetic nanoparticles (MNPs) of $25 \mathrm{~nm}$ size. The different amounts of MNP uptake is a characteristic of each neural cell type. to label mesenchymal stem cells from umbilical cord blood (UC-MSC) to evaluate the proliferation kinetics by MRI. ${ }^{24}$ Furthermore, MNPs have been used as a magnetic carrier to cross the blood brain barrier (BBB), a protective membrane of the neural tissue from toxins, alien substances, and sudden changes in blood composition. Indeed, by functionalizing NPs with brain-derived neurotrophic factor (BDNF), it has been possible to demonstrate the efficacy and ability of these nanovectors to cross the BBB for use as neuroprotectors. ${ }^{25}$

\section{Impact of iron-oxide MNPs on neural cells and the nervous system}

The effects of biological media on iron-oxide MNPs

When NPs come in contact with biological fluids, they adsorb proteins and other biomolecules. The formation of NP-protein complexes is commonly referred to as the NP-protein corona that can critically affect the interaction of NPs with living systems. ${ }^{26}$ The proteins adsorbed onto NPs depend on many variables, including the biological medium and the physicochemical characteristics of the NPs (see the Fadeel et al. article in this issue). ${ }^{27}$

There is consensus that the protein corona can influence the uptake of MNPs by cells, as well as their distribution and cytotoxicity. Moreover, this protein layer can modify the clearance, activity, and toxicity of MNPs through modifications in hydrodynamic size and surface charge. ${ }^{28}$ Thus, the protein-NP interaction mechanisms must be known in order to efficiently design MNPs that can target specific cell types. ${ }^{29}$

Functionalization of NPs with appropriate polymers that provide appropriate functional groups for attachment of specific biological molecules, such as neurotrophic factors or cell recognition moieties, is important in the design of NPs for biomedical applications. ${ }^{30,31}$ For instance, it has been recently shown that $\mathrm{Fe}_{3} \mathrm{O}_{4}$-based MNPs of similar size but with a different surface polymer coating (e.g., polyethyleneimine [PEI-MNPs] and poly(acrylic acid)-[PAA-MNPs]) strongly influence MNPs uptake by SH-SY5Y neuroblastoma cells, a tumor cell line of neural crest cell origin and an accepted model of neuronal cell behavior. The study concluded that PEI-MNPs - that adsorbed larger amounts of proteins compared to PAA-MNPs - were internalized by the cells in a higher extent compared to PAA-MNPs. ${ }^{28}$

The influence of MNP final properties on the internalization mechanism and resulting intracellular distribution can be studied by scanning transmission electron microscopy that provides images with $Z$-dependent intensity contrast $(Z$ being the atomic number of each element) so localization of MNPs within organelles is facilitated. Application of this technique to the study of internalization pathways for different cell types is shown in Figure 2 for PEI-MNPs and PAA-MNPs. It can be seen 

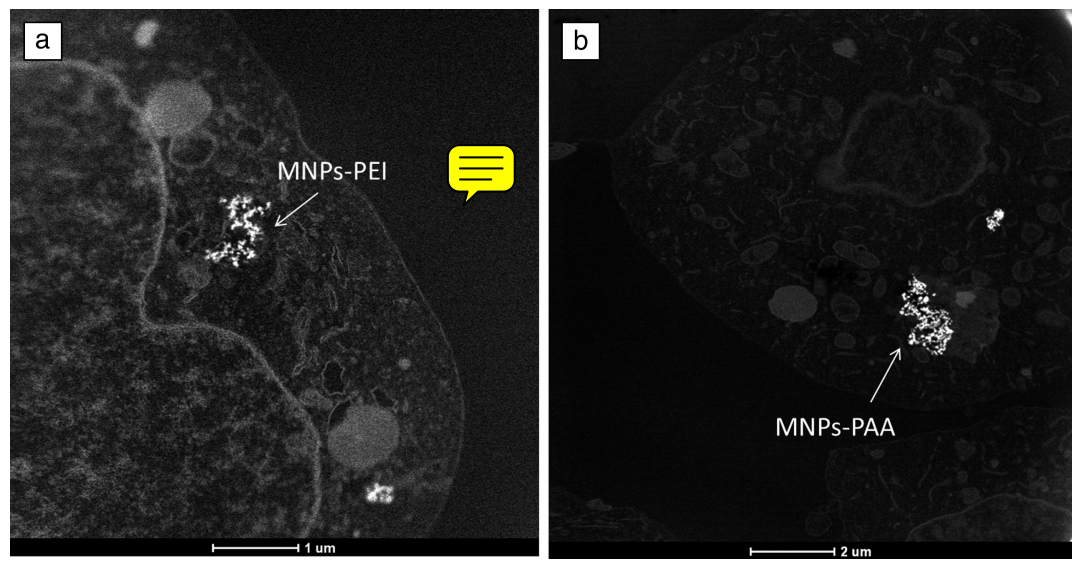

\section{Conclusions}

It is evident that magnetic nanoparticles are just beginning to reveal their potential as non-invasive agents for local actuation in different neurotherapies. The first steps toward cell targeting using specific surface molecules have been realized, and now "fine tuning" of the surface chemistry is necessary in order to promote axon regeneration. Further progress on materials functionalization is also required for a more efficient targeting of injured neural tissue, a pre-requisite for any magnetically guided regeneration therapy. Although re-myelinating axons and removing inhibitory factors are among the major problems yet

Figure 2. Scanning transmission electron microscopy high-angle annular dark-field images of SH-SY5Y cells incubated with (a) polyethyleneimine (PEI)-magnetic nanoparticles (MNPs) (Scale bar: $[\bar{\equiv}$ ) and (b) poly(acrylic acid)(PAA)-MNPs (Scale bar: $2 \mu \mathrm{m})$. to be solved, there is clear advancement on strategies for non-invasive guiding of neural cells and axonal regeneration. From a clinical applications perspective, much of the in vitro

that the intracellular distributions are different in the two cases due to the different protein corona compositions. ${ }^{26}$ It is clear that MNP surface properties dictate the major biological properties and consequently the interaction with a given cell type. Understanding the dynamics of this complex interaction is now recognized as the central requisite to better understand the biological impact of these novel materials on living organisms.

\section{Magnetically assisted neural growth and protection}

The importance of mechanical factors for the nervous system has been appreciated only recently. The idea of tension being involved in the morphogenesis of the nervous system arose in the late $1970 \mathrm{~s}$, when experimental pioneering work revealed that neuronal processes of growing neurons in vitro are under tension. ${ }^{32}$ Later, it was demonstrated that the external application of mechanical tension by glass needles alone is sufficient to initiate axonal outgrowth (i.e., axonal sprouting and lengthening) ${ }^{33}$ There is thus some evidence that mechanical tension can even induce "stretch growth" of axons, and this mechanism can be exploited therapeutically. ${ }^{34}$

Recently, Fass et al. ${ }^{35}$ reported the use of magnetic beads to apply a tensile force and study its effect on neurite elongation. They were able to identify a force threshold of $15-100 \mathrm{pN}$. In addition to the evidence that mechanical tension can induce "stretch growth" of neurites or process initiation, its influence on axonal re-growth orientation has also been investigated. Recently, we reported the use of a MNP-based technique for the application of very low tension values ( $\mathrm{pN}$ or below) on neuronal processes. ${ }^{7}$ This study demonstrated, using the rat pheochromocytoma PC12 cell line, PC12 cellline-that MNPs can be used to manipulate axons/neurites using external magnetic forces for controlled directional growth. Altogether, the existing literature indicates that forces generated by MNPs under static magnetic fields, when carefully controlled, could act as powerful stimulants of neuronal growth and development. proofs-of-concept are yet to be translated to the in vivo field in order to obtain the final goal (i.e., to achieve full neuronal regeneration and restoration of lost neuronal functions after injury), allowing patients to recover both motor and physiological functions.

\section{References}

1. J. Pinkernelle, P. Calatayud, G.F. Goya, H. Fansa, G. Keilhoff, BMC Neurosci. 13, 32 (2012).

2. W. Daly, L. Yao, D. Zeugolis, A. Windebank, A. Pandit, J. R. Soc. Interface 9, 202 (2012).

3. M. Siemionow, G. Brzezicki, Int. Rev. Neurobiol. 87, 141 (2009).

4. B.J. Dickson, Science 298, 1959 (2002)

5. T.S. Wong, S.H. Kang, S.K.Y. Tang, E.J. Smythe, B.D. Hatton, A. Grinthal, J. Aizenberg, Nature 477, 443 (2011).

6. Y. Yan, G.K. Such, A.P.R. Johnston, J.P. Best, F. Caruso, ACS Nano 6, 3663 (2012) 7. C. Riggio, M.P. Calatayud, M. Giannaccini, B. Sanz, T.E. Torres, R. FernandezPacheco, A. Ripoli, M.R. Ibarra, L. Dente, A. Cuschieri, G.F. Goya, V. Raffa, Nanomed. Nanotechnol. Biol. Med. (2014) (forthcoming).

8. D.A. Borton, M. Yin, J. Aceros, A. Nurmikko, J. Neural Eng. 10, 026010 (2013). 9. D. Kilinc, A. Blasiak, James J. O'Mahony, D.M. Suter, G.U. Lee, Biophys. J. 103, 1120 (2012)

10. J. Liu, J. Shi, L. Jiang, F. Zhang, L. Wang, S. Yamamoto, M. Takano, M. Chang, H. Zhang, Y. Chen, Appl. Surf. Sci. 258, 7530 (2012).

11. V. Zablotskii, T. Syrovets, Z.W. Schmidt, A. Dejneka, T. Simmet, Biomaterials 35, 3164 (2014).

12. K. Yue, R. Guduru, J. Hong, P. Liang, M. Nair, S. Khizroev, PLoS One 7, e44040 (2012)

13. A.P. Alivisatos, A.M. Andrews, E.S. Boyden, M. Chun, G.M. Church, K. Deisseroth, J.P. Donoghue, S.E. Fraser, J. Lippincott-Schwartz, L.L. Looger, S. Masmanidis, P.L. McEuen, A.V. Nurmikko, H. Park, D.S. Peterka, C. Reid, M.L. Roukes, A. Scherer, M. Schnitzer, T.J. Sejnowski, K.L. Shepard, D. Tsao, G. Turrigiano, P.S. Weiss, C. Xu, R. Yuste, X. Zhuang, ACS Nano 7, 1850 (2013). 14. P.K. Lakshmi, T.K. Indira, IJPSN 3,8 (2010).

15. I. Marcos-Campos, L. Asín, T.E. Torres, C. Marquina, A. Tres, M.R. Ibarra, G.F. Goya, Nanotechnology 22, 13 (2011).

16. P. Tartaj, M. Morales, S. Veintemillas-Verdaguer, T. González-Carreño, C.J. Serna, J. Phys. D: Appl. Phys. 36, R182 (2003).

17. S. Laurent, D. Forge, M. Port, A. Roch, C. Robic, L.V. Elst, R.N. Muller, Chem. Rev. 108, 2064 (2008).

18. J.K. Oh, J.M. Park, Prog. Polym. Sci. 36, 168 (2011).

19. N. Mizutani, T. Iwasaki, S. Watano, T. Yanagida, T. Kawai, Curr. Appl. Phys. 10, 801 (2010)

20. F.M. Kievit, O. Veiseh, N. Bhattarai, C. Fang, J.W. Gunn, D. Lee, L.G. Ellenbogen J.M. Olson, M. Zhang, Adv. Funct. Mater. 19, 2244 (2009).

21. C. Riggio, M. Pilar Calatayud, C. Hoskins, J. Pinkernelle, B. Sanz, T. Enrique Torres, M. Ricardo Ibarra, L. Wang, G. Keilhoff, G. Fabian Goya, V. Raffa, A. Cuschieri, Int. J. Nanomed. 7, 3155 (2012) 
22. M.P. Calatayud, C. Riggio, V. Raffa, B. Sanz, T.E. Torres, M.R. Ibarra, C. Hoskins, A. Cuschieri, L. Wang, J. Pinkernelle, G. Keilhoff, G.F. Goya, J. Mater. Chem. B1, 3607 (2013).

23. C. Riggio, S. Nocentini, M.P. Catalayud, G.F. Goya, A. Cuschieri, V. Raffa, J.A. del Río, Int. J. Mol. Sci. 14, 10852 (2013).

24. T.T. Sibov, L.F. Pavon, L.A. Miyaki, J.B. Mamani, L.P. Nucci, L.T. Alvarim, P.H. Silveira, L.C. Marti, L. Gamarra, Int. J. Nanomed. 9, 337 (2013).

25. S. Pilakka-Kanthikeel, V.S.R. Atluri, V. Sagar, S.K. Saxena, M. Nair, PLoS One 8, e62241 (2013).

26. S. Tenzer, D. Docter, J. Kuharev, A. Musyanovych, V. Fetz, R. Hecht, F. Schlenk, D. Fischer, K. Kiouptsi, C. Reinhardt, K. Landfester, H. Schild, M. Maskos, S.K. Knauer, R.H. Stauber, Nat. Nanotechnol. 8 (10), 772 (2013).
27. S.R. Saptarshi, A. Duschl, A.L. Lopata, J. Nanobiotechnology 11, 26 (2013). 28. M.P. Calatayud, B. Sanz, V. Raffa, C. Riggio, M.R. Ibarra, G.F. Goya, Biomaterials 35, 6389 (2014).

29. D. Eberbeck, M. Kettering, C. Bergemann, P. Zirpel, I. Hilger, L. Trahms, J. Phys. D: Appl. Phys. 43 (40), 405002 (2010).

30. M.L. Hilda T.R. Wiogo, V. Bulmus, L. Gutiérrez, R.C. Woodward, Langmuir 28, 4346 (2012).

31. N. Welsch, Y. Lu, J. Dzubiella, M. Ballauf, Polymer 54, $2835 \mathrm{e} 2849$ (2013).

32. D. Bray, J. Cell Sci. 37, 391 (1979).

33. D. Bray, Dev. Biol. 102, 379 (1984)

34. D.H. Smith, Prog. Neurobiol. 89, 231 (2009).

35. J.N. Fass, D.J. Odde, Biophys. J. 85, 623 (2003). 


\section{AUTHOR QUERIES}

QA $\quad$ The distinction between surnames can be ambiguous, therefore to ensure accurate tagging for indexing purposes online (eg for PubMed entries), please check that the highlighted surnames have been correctly identified, that all names are in the correct order and spelt correctly. 